\title{
Arithmetic properties of Ramanujan's general partition function for modulo 11
}

\author{
Belakavadi R. Srivatsa Kumar ${ }^{1, *}$, Ramakrishna Narendra², Karpenahalli R. Rajanna ${ }^{2}$ \\ ${ }^{1}$ Dept. of Mathematics, Manipal Institute of Technology \\ Manipal Acedemy of Higher Education, Manipal - 576 104, India \\ ${ }^{2}$ Dept. of Mathematics, Acharya Institute of Technology \\ Soladevanahalli, Bengaluru - 560 107, India \\ *Corresponding author: sri_vatsabr@yahoo.com
}

\begin{abstract}
In the present work, for the general partition function $p_{r}(n)$, we establish five new infinite families of congruences modulo 11. Our emphasis throughout this paper is to exhibit the use of $q$-identities to generate congruences of $p_{r}(n)$.
\end{abstract}

Keywords: Congruences; General partitions; $q$-identities; Theta-functions.

Mathematical Subject Classification 2010: Primary 11P83; Secondary 05A15, 05A17

\section{Introduction}

For $|x y|<1$, Ramanujan's general theta function $f(x, y)$ is given by

$f(x, y):=\sum_{k=-\infty}^{\infty} x^{\frac{k(k+1)}{2}} y^{\frac{k(k-1)}{2}}$.

The function $f(a, b)$ enjoys the well-known Jacobi triple product identity (Berndt 1991, p.35),

$f(x, y)=(-x ; x y)_{\infty}(-y ; x y)_{\infty}(x y ; x y)_{\infty}$,

where, here and throughout the paper, we will utilize the following $q$-shifted factorial and always assume $|q|<1$.

$(x ; q)_{\infty}=\prod_{k=0}^{\infty}\left(1-x q^{k}\right)$.

One of the special cases of $f(x, y)$ as defined by $\mathrm{S}$. Ramanujan (Berndt 1991) is as follows:

$$
\begin{aligned}
f(-q):=f\left(-q,-q^{2}\right) & =\sum_{n=-\infty}^{\infty}(-1)^{n} q^{\frac{n(3 n-1)}{2}} \\
& =(q ; q)_{\infty} .
\end{aligned}
$$

For convenience, we write $f_{n}=f\left(-q^{n}\right)$. Due to Euler, we have

$$
\sum_{n=0}^{\infty} p(n) q^{n}=\frac{1}{f_{1}}
$$

where $p(n)$ is the number of partitions of $n$. S. Ramanujan initiated the general partition function $p_{r}(n)$ as

$$
\sum_{n=0}^{\infty} p_{r}(n) q^{n}=f_{1}^{r}
$$

for non-zero integer $r$. For partition function $p(n)$, Ramanujan's so called "most beautiful identity" is given by

$$
\sum_{n=0}^{\infty} p(5 n+4) q^{n}=5 \frac{f_{5}^{5}}{f_{1}^{6}},
$$

which readily implies

$p(5 n+4) \equiv 0 \quad(\bmod 5)$.

The generalization of the congruences modulo powers of 5 and 7 for all $p_{r}(n)$ was proved by Ramanathan (1950). Later Atkin (1968) found that Ramanathan's proof is not correct. Further Newmann (1955, 1957a, 1957b) studied the function $p_{r}(n)$ and obtained several interesting congruences and identities involving $p_{r}(n)$. The functions $p_{r}(n)$ have been studied by many mathematicians. For the wonderful work one can see Atkin (1968), Baruah and Ojah (2011), Baruah and Sarmah (2013), Boylon (2004), Farkas and Kra (1999), Gandhi (1963), Gordon (1983), Kimming and Olsson (1992), and Saikia and Chetry (2018). Recently, Hammond and Lewis (2004) proved that

$p_{-2}(5 n+\ell) \equiv 0 \quad(\bmod 5)$, 
where $\ell \in\{2,3,4\}$. Also Chen et al. (2014) proved

$p_{-2}(25 n+23) \equiv 0 \quad(\bmod 25)$

by using modular forms. More recently, Tang (2018) proved some congruences modulo powers of 5 for $p_{r}(n)$ with $r \in\{2,6,7\}$. For example,

$$
\begin{aligned}
& p_{-2}\left(5^{2 \delta-1} n+\frac{7 \times 5^{2 \delta-1}+1}{12}\right) \\
\equiv & p_{-6}\left(5^{2 \delta} n+\frac{3 \times 5^{\delta}+1}{4}\right) \\
\equiv & p_{-7}\left(5^{2 \delta-1} n+\frac{13 \times 5^{2 \delta-1}+7}{24}\right) \\
\equiv & 0 \quad\left(\bmod 5^{\delta}\right) .
\end{aligned}
$$

Motivated by the above work, we deduce new infinite families of congruences modulo 11 for $p_{r}(n)$ by using $q$-identities for any positive integer $\lambda$. The results in this paper are given below:

Theorem 1.1 If $\tau=3,6,8,9,10$, then

$p_{11 \lambda+1}(11 n+\tau) \equiv 0 \quad(\bmod 11)$.

Theorem 1.2 If $\tau=2,4,5,7,8,9$, then

$p_{11 \lambda+3}(11 n+\tau) \equiv 0 \quad(\bmod 11)$.

Theorem 1.3 We have

$p_{11 \lambda+6}(11 n+8) \equiv 0 \quad(\bmod 11)$.

Theorem 1.4 If $1 \leq \tau \leq 10$, then

$p_{121 \lambda+1}(121 n+11 \tau+5) \equiv 0 \quad(\bmod 11)$.

Theorem 1.5 If $1 \leq \tau \leq 10$, then

$p_{121 \lambda+2}(121 n+11 \tau+10) \equiv 0 \quad(\bmod 11)$.

\section{Proofs of Theorems $1.1-1.5$}

Proof of Theorem 1.1: Setting $r=11 \lambda+1$ in (1), we have

$\sum_{n=0}^{\infty} p_{11 \lambda+1}(n) q^{n}=f_{1}^{11 \lambda+1}=f_{1}^{11 \lambda} f_{1}$.

From the binomial theorem, it follows that

$f_{1}^{11} \equiv f_{11} \quad(\bmod 11)$

Substituting (3) into (2), we see that $\sum_{n=0}^{\infty} p_{11 \lambda+1}(n) q^{n} \equiv f_{11}^{\lambda} f_{1} \quad(\bmod 11)$

From Bernd (1991, p. 363, Entry 6(iii)), we have

$$
\begin{aligned}
& f_{1}=f_{121}\left(A\left(q^{11}\right)-q B\left(q^{11}\right)-q^{2} C\left(q^{11}\right)\right. \\
& \left.+q^{5}+q^{7} D\left(q^{11}\right)-q^{15} E\left(q^{11}\right)\right)
\end{aligned}
$$

where

$$
\begin{aligned}
& A:=A\left(q^{11}\right)=\frac{f\left(-q^{44}, q^{77}\right)}{f\left(-q^{22},-q^{99}\right)}, \\
& B:=B\left(q^{11}\right)=\frac{f\left(-q^{22}, q^{99}\right)}{f\left(-q^{11},-q^{110}\right)},
\end{aligned}
$$

$C:=C\left(q^{11}\right)=\frac{f\left(-q^{55}, q^{66}\right)}{f\left(-q^{33},-q^{88}\right)}$,

$D:=D\left(q^{11}\right)=\frac{f\left(-q^{33}, q^{88}\right)}{f\left(-q^{44},-q^{77}\right)}$

and

$E:=E\left(q^{11}\right)=\frac{f\left(-q^{11}, q^{110}\right)}{f\left(-q^{55},-q^{66}\right)}$.

Invoking (5) in (4), it is observed that

$\sum_{n=0}^{\infty} p_{11 \lambda+1}(n) q^{n} \equiv f_{11}^{\lambda} f_{121}\left(A-q B-q^{2} C\right.$

$\left.+q^{5}+q^{7} D-q^{15} E\right) \quad(\bmod 11)$.

Selecting the terms containing $q^{11 n+\tau}$ for $\tau=3,6,8,9,10$ on both sides of (6), we obtain the required congruence.

Proof of Theorem 1.2: Setting $r=11 \lambda+3$ in (1) and then using (3), we obtain

$$
\begin{aligned}
\sum_{n=0}^{\infty} p_{11 \lambda+3}(n) q^{n} & =f_{1}^{11 \lambda+3}=f_{1}^{11 \lambda} f_{1}^{3} \\
& \equiv f_{11}^{\lambda} f_{1}^{3}(\bmod 11) .
\end{aligned}
$$

From Berndt (1991, p. 39, Entry 24(ii)), we have

$f_{1}^{3}=\sum_{n=0}^{\infty}(-1)^{n}(2 n+1) q^{n(n+1) / 2}$,

and it follows that 


$$
\begin{aligned}
f_{1}^{3} & =I_{0}\left(q^{11}\right)-3 q I_{1}\left(q^{11}\right)+5 q^{3} I_{2}\left(q^{11}\right) \\
& -7 q^{6} I_{3}\left(q^{11}\right)+9 q^{10} I_{4}\left(q^{11}\right)-11 q^{15} I_{5}\left(q^{11}\right) \\
& \equiv I_{0}\left(q^{11}\right)-3 q I_{1}\left(q^{11}\right)+5 q^{3} I_{2}\left(q^{11}\right) \\
& -7 q^{6} I_{3}\left(q^{11}\right)+9 q^{10} I_{4}\left(q^{11}\right) \quad(\bmod 11)
\end{aligned}
$$

where $I_{0}, I_{1}, I_{2}, I_{3}$ and $I_{4}$ are the series with integral powers of $q^{11}$ : Invoking (9) in (7), we have

$\sum_{n=0}^{\infty} p_{11 \lambda+3}(n) q^{n} \equiv f_{11}^{\lambda}\left(I_{0}-3 q I_{1}+5 q^{3} I_{2}\right.$

$\left.-7 q^{6} I_{3}+9 q^{10} I_{4}\right) \quad(\bmod 11)$.

Selecting the terms containing $q^{11 n+\tau}$ for $\tau=2,4$, $5,7,8,9$ on both sides of (10), we obtain the required congruence.

Proof of Theorem 1.3: Setting $r=11 \lambda+6$ in (1) and then using (3), we obtain

$\sum_{n=0}^{\infty} p_{11 \lambda+6}(n) q^{n} \equiv f_{11}^{\lambda} f_{1}^{6} \quad(\bmod 11)$.

On squaring (9) and then grouping, we deduce

$$
\begin{aligned}
f_{1}^{6} \equiv & I_{0}^{2}\left(q^{11}\right)+I_{1}\left(q^{11}\right) I_{4}\left(q^{11}\right)+5 q\left(I_{0}\left(q^{11}\right)\right. \\
& \left.\times I_{1}\left(q^{11}\right)+I_{3}^{2}\left(q^{11}\right)\right)+q^{2}\left(9 I_{1}^{2}\left(q^{11}\right)\right. \\
& \left.+2 I_{2}\left(q^{11}\right) I_{4}\left(q^{11}\right)\right)+10 q^{3} I_{0}\left(q^{11}\right) I_{2}\left(q^{11}\right) \\
& +3 q^{4} I_{1}\left(q^{11}\right) I_{2}\left(q^{11}\right)+6 q^{5} I_{3}\left(q^{11}\right) I_{4}\left(q^{11}\right) \\
& +q^{6}\left(8 I_{0}\left(q^{11}\right) I_{3}\left(q^{11}\right)+3 I_{2}^{2}\left(q^{11}\right)\right) \\
& +9 q^{7} I_{1}\left(q^{11}\right) I_{3}\left(q^{11}\right)+q^{9}\left(7 I_{2}\left(q^{11}\right) I_{3}\left(q^{11}\right)\right. \\
& \left.+4 I_{4}^{2}\left(q^{11}\right)\right)+7 q^{10} I_{0}\left(q^{11}\right) I_{4}\left(q^{11}\right) \\
& (\bmod 11),
\end{aligned}
$$

Invoking (12) in (11), we have

$$
\begin{aligned}
\sum_{n=0}^{\infty} p_{11 \lambda+6}(n) q^{n} \equiv f_{11}^{\lambda}\left(I_{0}^{2}+I_{1} I_{4}\right. \\
\quad+5 q\left(I_{0} I_{1}+I_{3}^{2}\right)+q^{2}\left(9 I_{1}^{2}+2 I_{2} I_{4}\right) \\
\quad+10 q^{3} I_{0} I_{2}+3 q^{4} I_{1} I_{2}+6 q^{5} I_{3} I_{4} \\
\quad+q^{6}\left(8 I_{0} I_{3}+3 I_{2}^{2}\right)+9 q^{7} I_{1} I_{3}+q^{9}\left(7 I_{2} I_{3}\right. \\
\left.\left.+4 I_{4}^{2}\right)+7 q^{10} I_{0} I_{4}\right) \quad(\bmod 11) .
\end{aligned}
$$

Selecting the terms containing $q^{11 n+8}$ on both sides of (13), we obtain the required congruence.
Proof of Theorem 1.4: Setting $r=121 \lambda+1$ in (1) and then using (3), we obtain

$\sum_{n=0}^{\infty} p_{121 \lambda+1}(n) q^{n} \equiv f_{121}^{\lambda} f_{1} \quad(\bmod 11)$

Invoking (5) in (14), we have

$\sum_{n=0}^{\infty} p_{121 \lambda+1}(n) q^{n} \equiv f_{121}^{\lambda+1}\left(A-q B-q^{2} C\right.$

$\left.+q^{5}+q^{7} D-q^{15} E\right) \quad(\bmod 11)$.

Selecting the terms containing $q^{11 n+5}$ on both sides of (15), dividing by $q^{5}$ and then letting $q$ to $q^{1 / 11}$, we obtain

$\sum_{n=0}^{\infty} p_{121 \lambda+1}(11 n+5) q^{n} \equiv f_{11}^{\lambda+1} \quad(\bmod 11)$.

Selecting the terms containing $q^{11 n+\tau}$ for $1 \leq \tau \leq 10$ on both sides of (16), we obtain the required congruence.

Proof of Theorem 1.5: Setting $r=121 \lambda+2$ in (1) and then employing (3), we have

$\sum_{n=0}^{\infty} p_{121 \lambda+2}(n) q^{n} \equiv f_{121}^{\lambda} f_{1}^{2} \quad(\bmod 11)$.

Squaring (5), we find that

$$
\begin{aligned}
f_{1}^{2} & =f_{121}^{2}\left(A^{2}-2 q A B+q^{2}\left(B^{2}-2 A C\right)\right. \\
& +2 q^{3} B C+q^{4} C^{2}+2 q^{5} A-2 q^{6} B+q^{7} \\
& \times 2(A D-C)-2 q^{8} B D-2 q^{9} C D+q^{10} \\
& +2 q^{12} D+q^{14} D^{2}-2 q^{15} A E+2 q^{16} B E \\
& \left.+2 q^{17} C E-2 q^{20} E-2 q^{22} D E+q^{30} E^{2}\right) .
\end{aligned}
$$

Invoking (18) in (17), it is observed that

$$
\begin{aligned}
\sum_{n=0}^{\infty} p_{121 \lambda+2}(n) q^{n} \equiv f_{121}^{\lambda+2}\left(A^{2}-2 q A B\right. \\
\quad+q^{2}\left(B^{2}-2 A C\right)+2 q^{3} B C+q^{4} C^{2} \\
\quad+2 q^{5} A-2 q^{6} B+2 q^{7}(A D-C)-2 q^{8} \\
\quad \times B D-2 q^{9} C D+q^{10}+2 q^{12} D+q^{14} D^{2} \\
\quad-2 q^{15} A E+2 q^{16} B E+2 q^{17} C E-2 q^{20} E \\
\left.\quad-2 q^{22} D E+q^{30} E^{2}\right) \quad(\bmod 11) .
\end{aligned}
$$

Selecting the terms containing $q^{121 \lambda+10}$ on both sides, dividing throughout by $q^{10}$ and letting $q$ by $q^{1 / 11}$, we deduce that 
$\sum_{n=0}^{\infty} p_{121 \lambda+2}(121 n+10) q^{n} \equiv f_{11}^{\lambda+2}(\bmod 11)$

Selecting the terms containing $q^{121 \lambda+10}$ for $1 \leq \tau \leq 10$ on both sides of (20), we obtain the required congruence.

\section{ACKNOWLEDGMENTS}

The authors would like to thank the anonymous referees for helpful suggestions and comments, which have greatly improved the original version of the paper. The first author thanks SERB, DST, India, for sanctioning the project [EMR/2016/001601]. Authors would like to thank Mr. Vinay Madhsudanan, MIT, Manipal, for helping during the preparation of final copy of the Latex file.

\section{References}

Andrews, G.E. (1976). The Theory of Partitions. Encyclopedia of Mathematics and its Applications, Addison-Wesley, Reading. (Reprinted: Cambridge Univ. Press, London and New York, 1998).

Atkin, A.O.L. (1968). Ramanujan congruences for $p_{k}(n)$, Canadian Journal Mathematics 20:67-78.

Baruah, N.D. \& Ojah, K.K. (2011). Some congruences deducible from Ramanujan's cubic continued fraction, International Journal Number Theory 7:1331-134.

Baruah, N.D. \& Sarmah, B.K. (2013). Identities and congruences for the general partition and Ramanujan $\tau$ functions, Indian Journal of Pure and Applied Mathematics 44(5):643-671.

Berndt, B.C. (1991). Ramanujan's Notebooks, Part III. New York, Springer.

Boylan, M. (2004). Exceptional congruences for powers of the partition functions, Acta Arithmetica 111:187-203.

Chen, W.Y.C., Du, D.K., Hou, Q.H. \& Sun, L.H. (2014). Congruences of multi-partition functions modulo powers of primes, Ramanujan Journal 35:1-19.

Farkas, H.M. \& Kra, I. (1999). Ramanujan Partition identities, Contemporary Mathematics 240:111-130.

Gandhi, J.M. (1963). Congruences for $p_{k}(n)$ and Ramanujan's $\tau$ function, American Mathematical Monthly 70:265-274.

Gordon, B. (1983). Ramanujan congruences for $p_{k}$ $\left(\bmod 11^{r}\right)$, Glasgow Mathematical Journal 24:107-123.

Hammond, P. \& Lewis, R. (2004). Congruences in ordered pairs of partitions, International Journal of Mathematics and Mathematical Sciences, 45-48:25092512.

Kiming, I. \& Olsson, J.B. (1992). Congruences like Ramanujan's for powers of the partition function, Archiv der Mathematik (Basel) 59(4):348-360.

Newmann, M. (1955). An identity for the coefficients of certain modular forms, Journal of London Mathematics Society, 30:488-493.

Newmann, M. (1957a). Some theorems about $p_{k}(n)$, Canadian Journal Mathematics 9:68-70.

Newmann, M. (1957b). Congruence for the coefficients of modular forms and some new congruences for the partition function, Canadian Journal of Mathematics 9:549-552.

Ramanathan, K.G. (1950). Identities and congruences of the Ramanujan type, Canadian Journal of Mathematics 2:168-178.

Ramanujan, S. (1919). Some properties of $p(n)$ the number of partitions of $n$, Mathematical Proceedings of Cambridge Philosophical Society, 19:207-210.

Ramanujan, S. (1920). Congruence properties of partitions, Proceedings of London Mathematical Society 18:Records of 13 March 1919.

Ramanujan, S. (1921). Congruence properties of partitions, Math. Zelt 9:147-153.

Saikia, N. \& Chetry, J. (2018). Infinite families of congruences modulo 7 for Ramanujan's general partition function, Annales Mathematiques du Quebec 42(1):127132.

Tang, D. (2018). Congruences modulo powers of 5 for $k$-colored partitions, Journal Number Theory, 187:198214.

Submitted : 13/12/2019

Revised : 08/07/2020

Accepted : 16/07/2020

DOI $\quad: 10.48129 /$ kjs.v48i1.8827 